their consequences. This study assessed unintended pregnancy and its associated factors among female sex workers in Northern Ethiopia.

Methods A community based cross-sectional study was conducted among 346 female sex workers at five localities of Mekelle city from March to April, 2014. Sex workers were selected with simple random sampling technique using sampling frame obtained from urban health extension program. Epi-data version 3.1 was used to enter data and analysis was done using SPSS version 20. Bivariate and multivariate logistic regressions were performed to identify factors associated with unintended pregnancy using odds ratio and 95\% confidence interval with P-value of 0.05 .

Results The magnitude of unintended pregnancy among female sex workers was $28.6 \%$. During this period, 59 women had abortion which represents three-fifths, (59.6\%) of those with unintended pregnancy. Female sex workers who had history of abortion formerly had 15.6 (AOR $=15.64$ 95\% CI: [8.03, 30.47]) times higher odds of unintended pregnancy compared to their counterparts. Sex workers who had steady partners had $2.9(\mathrm{AOR}=2.87,95 \% \mathrm{CI}:[1.47,5.61])$ times higher odds of have unintended pregnancy than those who hadn't. Drug users had 2.7 (AOR $=2.68,95 \% \mathrm{CI}$ : [1.30, 5.52]) times higher odds of unintended pregnancy than those who hadn't use. Sex workers who had longer duration in sex work were $67 \%$ less likely to have unintended pregnancy than those with $<12$ months (AOR $=0.33$, 95\% CI: [0.11, 0.95]).

Conclusion High level of unintended pregnancy and a range of associated factors were identified among sex workers. Improving utilization of effective pregnancy prevention methods in a consistent manner can avert the existing high level of unintended pregnancy among female sex workers.

Disclosure No significant relationships.

\section{P701 TIME TRENDS IN PREVALENCE AND INCIDENCE OF HIV, GONORRHEA AND CHLAMYDIA AMONG FEMALE SEX WORKERS IN BENIN, 2008-2018}

${ }^{1}$ Michel Alary*, ${ }^{2}$ Luc Béhanzin, ${ }^{2}$ Fernand Guédou, ${ }^{2}$ Ella Goma-Matsétsé, ${ }^{2}$ Marlène AzaGnandji, ${ }^{3}$ Djimon Marcel Zannou, 'Souleymane Diabaté, ${ }^{3}$ Dissou Affolabi, ${ }^{4}$ René Kêkê, ${ }^{4}$ Moussa Bachabi, ${ }^{3}$ Flore Gangbo. ${ }^{1} \mathrm{CHU}$ de Quebec - Université Laval, Quebec, Canada; ${ }^{2}$ Dispensaire IST, Cotonou, Benin; 'Université d'Abomey-Calavi, Cotonou, Benin; ${ }^{4}$ Programme Santé de lutte contre le SIDA, Cotonou, Benin

\subsection{6/sextrans-2019-sti.767}

Background The conduction of 3 cohort studies among professional female sex workers (FSWs), e.g. women whose main source of revenue is sex work, since 2008 in Benin allowed the estimation of time trends in HIV, gonorrhea and chlamydia prevalence and incidence in this key population over the last decade.

Methods The 3 cohort studies were conducted from 09/200803/2012, 10/2014-12/2016 and 04/2017-09/2018, respectively. Women were recruited at the Dispensaire IST, a FSW-dedicated clinic, after providing informed consent. Follow-up was quarterly in the first 2 studies and bi-annual in the latest one. FSWs were tested for HIV (rapid test with confirmatory assay according to Benin guidelines), gonorrhea and chlamydia (BD ProbeTec $^{\mathrm{TM}} \mathrm{CT} / \mathrm{NG}$, Becton Dickinson Inc.) at baseline and at each follow-up for HIV and semi-annually for gonorrhea and chlamydia. Questionnaires were administered.
Results In all studies, median age was 32-33 years and the proportion of women not born in Benin 50-60\%. HIV [gonorrhea (chlamydia)] prevalence was $37.1 \%$ [4.3\% (3.5\%)] in $2008(\mathrm{n}=396), 29.1 \%$ [9.7\% (4.8\%)] in $2014(\mathrm{n}=361)$ and $26.0 \%$ [13.8\% (7.4\%)] in $2017(\mathrm{n}=312)$. There was a significant downward (upward) trend in HIV (gonorrhea and chlamydia) prevalence: $\mathrm{p}<0.001 \quad(\mathrm{p}<0.001$ and $\mathrm{p}=0.03)$. Among respectively 319, 299 and 213 women with follow-up, gonorrhea (chlamydia) incidence was 8.9 (2.1), 9.0 (4.6) and 14.8 (5.8) per 100 person-years; $p=0.07(p=0.003)$. HIV incidence was $1.4,0.8$ and 0.7 per 100 person-years in 2008-12, 2014-16 and 2017-18, respectively $(\mathrm{p}=0.41)$.

Conclusion HIV prevalence is decreasing, but still high, whereas HIV incidence is low in this highly mobile key population. This combination of high prevalence and low incidence is likely due to HIV treatment scale-up over the last decade, including the adoption of an HIV "test and treat" strategy since 2016 in Benin. However, the increase in both gonorrhea and chlamydia is worrying and calls for renewed control strategies for these curable infections.

Disclosure No significant relationships.

\section{P702 CHARACTERIZING HIV-ASSOCIATED VULNERABILITIES AMONG WOMEN ENGAGED IN TRANSACTIONAL SEX TO DESIGN PROGRAMS IN UKRAINE}

${ }^{1}$ Daryna Pavlova*, ${ }^{2}$ Olga Balakireva, ${ }^{3}$ Eve Cheuk, ${ }^{4}$ Shajy Isac, ${ }^{3}$ Robert Lorway, ${ }^{3}$ Michael Pickles, ${ }^{5}$ Paul Sandstrom, ${ }^{6}$ Sharmistha Mishra, ${ }^{3}$ James Blanchard, ${ }^{3}$ Marissa Becker. "NGO "Ukrainian Institute for Social Research after Oleksandr Yaremenko", Kyiv, Ukraine; ${ }^{2}$ Institute for Economics and Forecasting, Ukrainian National Academy of Sciences, Kyiv, Ukraine; ${ }^{3}$ Center for Global Public Health, Department of Community Health Sciences, University of Manitoba, Winnipeg, Canada; ${ }^{4}$ India Health Action Trust, Bangalore, India; ${ }^{5}$ National HIV and Retrovirology Laboratory, JC Wilt Infectious Diseases Research Centre, Winnipeg, Canada; ' ${ }^{6} i$ Ka Shing Knowledge Institute, St. Michael's Hospital, University of Toronto, Toronto, Canada

\subsection{6/sextrans-2019-sti.768}

Background Evidence suggests a range of HIV-associated vulnerabilities associated with the exchange of sex for money or other material goods, but most research and programs in Ukraine focus on formal sex work. We sought to describe the prevalence of HIV and HIV-associated vulnerabilities among adolescent girls and young women engaged in transactional sex outside of formal sex work.

Methods We conducted a cross-sectional bio-behavioral study in Dnipro, Ukraine in 2016 of young women (14-24 years). 469 participants were recruited who reported transactional sex but not sex work, from places where female sex workers solicit clients. Transaction sex was defined as a sexual engagement with the expectation of receiving, gifts or other resources in return, when the price of sex is often not negotiated upfront and is implicitly understood.

Results Mean age of participants was 21.2 years. The mean age among participants at first sex was 16.0 years (range 1221). At first sex $34 \%$ (160) received gifts or money, and $7 \%$ (34) reported forced first sex. During the past week, $81 \%$ (378) had sex with a regular transactional partner, with whom $35 \%$ (132) had condomless sex. In the past month, 29\% (137) had sex while inebriated with regular transactional partners, and 64\% (299) did not use/remember whether a 
condom was used during last sex act with them. 49\% (231) reported also having non-transactional intimate partners, with whom 46\% (107) reported condomless sex in the last week. $56 \%$ (262) never had an HIV test and $87 \%$ (408) were unaware of HIV prevention programs in Dnipro. HIV prevalence was $1.7 \%(8)$.

Conclusion The high prevalence of HIV-associated vulnerabilities, low testing rates, and high HIV prevalence highlight the importance of designing HIV prevention programs to reach young women engaging in transactional sex.

Disclosure No significant relationships.

\section{P703 PREGNANCY INTENTION AND PREVALENCE ACCORDING TO HIV STATUS AMONG FEMALE SEX WORKERS IN MALI}

${ }^{1}$ Gentiane Perrault Sullivan*, ${ }^{2}$ Nana Camara, ${ }^{3}$ Bintou Dembele, ${ }^{4}$ Fernand Guédou, ${ }^{3}$ Ismaila Thera, ${ }^{5}$ Fatoumata Korika Tounkara, ${ }^{6}$ Michel Alary. ' Laval University, Québec, Canada; ${ }^{2}$ ARCADSIDA, Bamako, Mali; ${ }^{3} A R C A D / S I D A$, Bamako, Mali; ${ }^{4}$ Dispensaire IST, Cotonou, Benin; ${ }^{5}$ Axe Santé des Populations et Pratiques Optimales en Santé, HSS, Social and Preventive Medicine, Québec, Canada; ${ }^{6} \mathrm{CHU}$ de Quebec - Université Laval, Quebec, Canada

\subsection{6/sextrans-2019-sti.769}

Background Women living in Sub-Saharan Africa have the world's highest rates of new HIV infections and unintended pregnancies. These two risks are magnified in the female sex worker's (FSW) population where HIV prevalence is 12 times higher than in the general population. Yet, no information is available concerning FSW's pregnancy intentions, which could help prevent HIV mother-to-child transmission and unintended pregnancies. This study investigated whether pregnancy intention and pregnancy prevalence varied according to FSWs' HIV status. We hypothesized that FSWs living with HIV (FSWLHIV) were less likely to intent carrying a pregnancy and to be pregnant.

Methods We analysed baseline data from a prospective observational cohort study. Three hundreds and twenty-five FSWs were recruited in Bamako, Mali (November 2017 - February 2018). Participants completed a questionnaire. We assessed pregnancy frequencies among women according to HIV status and compared those using chi-square. Age-adjusted odds ratios were estimated using logistic regression.

Results Mean age was 25 years $(\mathrm{N}=303), 20.8 \%$ of the participants were HIV positive and $16.2 \%$ had the intention of becoming pregnant during the next six months. The proportion of FSWs reporting having been pregnant prior to entering in sex work were $72.1 \%(44 / 61)$ for the FSWLHIV and $60.8 \%(142 / 240)$ the other FSWs (p-value=0.2452). The occurrence of pregnancies since engagement in sex work was reported much less often (FSWLHIV $=36.1 \%$ and others $=$ $28.8 \%$ ) with still no significant difference between the two groups ( $\mathrm{p}$-value $=0.3494$ ). FSWLHIV reported more frequently to have wanted a pregnancy in the last 6 months compared to other FSWs (aOR $=2.57,95 \% \mathrm{CI}$ : $[1.3,2.2])$.

Conclusion With FSWLHIV being more likely to desire children during sex work practice, while less than half of them currently receive ART, a specific attention should be given to support them in that decision in order to prevent mother-tochild transmission.

Disclosure No significant relationships.

\section{P705 BACTERIAL SEXUALLY TRANSMITTED INFECTIONS AMONG WOMEN WHO INJECT DRUGS AND EXCHANGE SEX IN KING COUNTY, WASHINGTON}

${ }^{1}$ Megan Curtis, ${ }^{2}$ Courtney Moreno, ${ }^{1}$ Lindley Barbee*, ${ }^{1}$ Sara Glick. ' University of Washington, Medicine, Seattle, USA; ${ }^{2}$ Public Health - Seattle and King County, Seattle, USA

\subsection{6/sextrans-2019-sti.770}

Background Women who inject drugs are at elevated risk for many adverse health outcomes, including sexually transmitted infections (STIs). Exchange sex is prevalent in this population, but its independent effect on STIs is unclear.

Methods We included data regarding the past year from cisgender women age $<60$ who reported injecting drugs and participated in the 2015 or 2016 cycles of the National HIV Behavioral Surveillance (NHBS) survey in Seattle $(\mathrm{N}=377)$. Using a log-binomial regression model, we estimated the odds of self-reported bacterial STI (gonorrhea, syphilis, or chlamydia) among women who inject drugs associated with exchanging sex for money or drugs. We adjusted for sociodemographic characteristics, condom use, and substance use behaviors. We hypothesized that engaging in exchange sex would be independently associated with increased odds of STI.

Results The period prevalence of bacterial STI in the past 12 months was $8.1 \%$ in the full sample. On univariate analysis, STI prevalence was significantly higher among women who reported exchange sex than among those who did not $(10.8 \%$ vs $4.0 \% ; \mathrm{p}=0.02)$ as was STI testing $(53.4 \%$ vs $34.0 \%$; $\mathrm{p}<0.01)$. Women who exchanged sex were less likely to report condomless vaginal or anal sex at last intercourse $(84.4 \%$ vs $89.1 \% ; p=0.19)$. Women who exchanged sex were more likely to use non-injection crack $(59.1 \%$ vs $38.8 \%$; $\mathrm{p}<0.01$ ) and were more likely to report heavy alcohol use (31.3\% vs $19.7 \%$; $=0.01)$. After adjusting for sociodemographic variables, condom use, and substance use; the association between exchange sex and STI remained statistically significant (adjusted odds ratio $=2.7 ; 95 \%$ confidence interval: 1.1-7.2)

Conclusion In this sample of Seattle area women who inject drugs, exchanging sex for drugs or money was associated with double the odds of STI which could be a result of more frequent STI testing. Healthcare providers should prioritize making testing and treatment accessible and appropriate for women who exchange sex.

Disclosure No significant relationships.

\section{P706 LOW GONORRHEA AND CHLAMYDIA TESTING RATE AMONG FEMALE SEX WORKERS IN GUANGDONG PROVINCE: A CROSS-SECTIONAL ANALYSIS}

${ }^{1}$ Peizhen Zhao*, ${ }^{2}$ Fan Yang, ${ }^{1}$ Yajie Wang, 'Weiming Tang, ${ }^{3}$ He-Ping Zheng, ${ }^{4}$ Bin Yang, ${ }^{1}$ Cheng Wang. 'Dermatology Hospital of Southern Medical University, Guangzhou, China; ${ }^{2}$ University of North Carolina Project - China, Guangzhou, China; ${ }^{3}$ Dermatology Hospital of Southern Medical University, Guangdong Center for STD Control and Prevention, Guangzhou, China; ${ }^{4}$ Dermatology Hospital, Southern Medical University, Guangzhou, China

\subsection{6/sextrans-2019-sti.771}

Background Female sex workers (FSW) are at high risk of gonorrhea and chlamydia infection. However, gonorrhea and chlamydia testing rate remain low in this population. This study aimed to assess the gonorrhea and chlamydia testing 